\title{
Koronarer Bypass besser als Katheterdilatation
}

Fragestellung: Ist die aortokoronare Bypass-Operation (CABG) auch bei Typ-1-Diabetikern mit koronarer Mehrgefäßerkrankung der perkutanen Katheterdilatation (PCI) überlegen?

Hintergrund: Inzwischen gibt es mehrere Studien, die bei Patienten mit stabiler koronarer Mehrgefäßerkrankung eine bessere Prognose für die Revaskularisierung mittels CABG im Vergleich zur PCI nachweisen. Deshalb empfehlen die Fachgesellschaften inzwischen die Bypass-Operation als bevorzugte $\mathrm{Me}$ thode. Das gilt insbesondere auch für Diabetiker, die $25 \%$ der Patienten mit Mehrgefäßerkrankung stellen und besonders häufig in randomisierten Studien untersucht wurden. Allerdings wurde in keiner dieser Studien zwischen Typ-1- und Typ-2-Diabetes unterschieden.

Patienten und Methoden: Deshalb wurden jetzt in einer Beobachtungsstudie alle Typ-1-Diabetiker analysiert, die zwischen 1995 und 2013 revaskularisiert wurden: 683 mittels CABG und 1.863 mittels PCI. Die Daten stammen aus nationalen, schwedischen Registern.

Ergebnisse: Nach einer Verlaufsdauer von 10,6 Jahren lag die relative Risikoreduktion bei allen spezifischen kardialen Endpunkten nach der CABG signifikant niedriger: Todesfälle infolge koronarer Herzkrankheit (-45\%), Herzinfarkte $(-47 \%)$
Originalie

Nyström T, Sartipy U, Franzén $S$ et al. PCI Versus CABG in Patients With Type 1 Diabetes and Multivessel Disease. J Am Coll Cardiol 2017;70:1441-51 und erneute Revaskularisierungen (-564\%). Allerdings war in der CABG-Gruppe die Gesamtmortalität mit $53 \%$ höher als in der PCI-Gruppe mit $45 \%$, doch verfehlte dieser Unterschied knapp die

Signifikanz (Risikozunahme um 1,14; 95\%-KI 0,99 bis 1,32). Bei Schlaganfällen und Herzinsuffizienz gab es keine Unterschiede.

Schlussfolgerung: Nach dieser Studie sind die bisherigen Ergebnisse zur Überlegenheit von CABG gegenüber PCI bei Diabetikern mit koronarer Mehrgefäßerkrankung auch auf den Typ-1-Diabetes übertragbar.

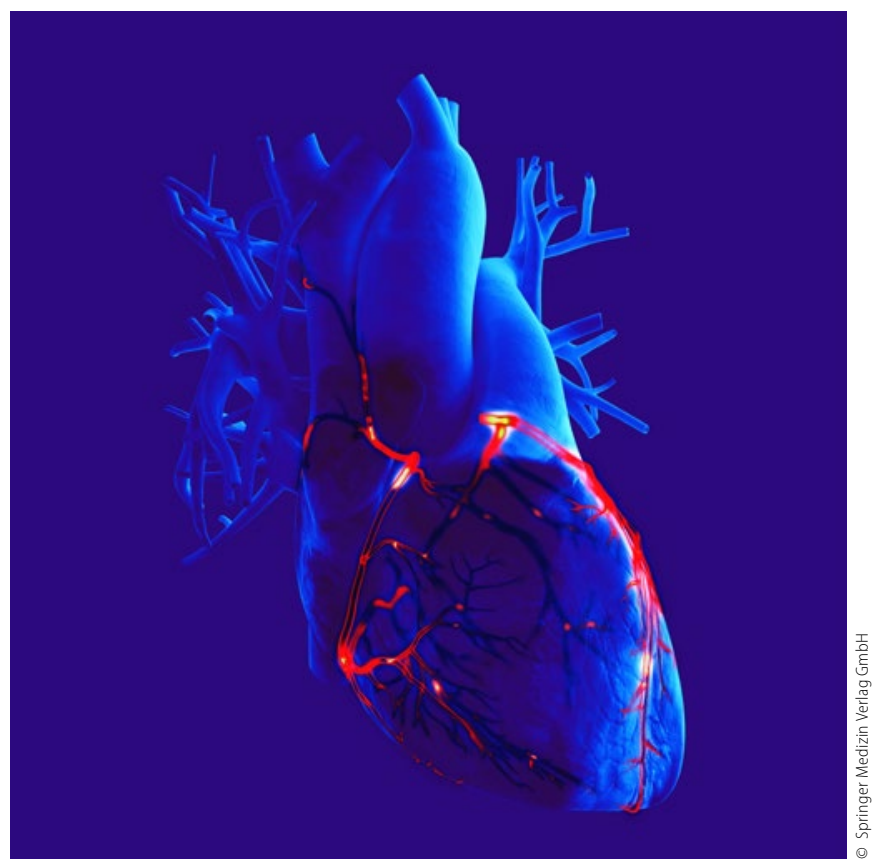

Die Fachgesellschaften werden bei koronarer Mehrgefäßerkrankung wohl weiterhin die CABG empfehlen, auch bei Typ-1-Diabetikern.

\section{- Kommentar von Prof. Dr. med. Heinrich Holzgreve}

\section{Datenfreie Zone beseitigt und Theorie erklärt Fakten}

Was die Größe der Erfolgsraten und die methodische Zuverlässigkeit angeht, erreicht diese Beobachtungstudie nicht ihre Vorgänger. Durch die Begrenzung auf Typ-1-Diabetiker bereichert sie allerdings eine bisher datenfreie Zone. Man kann davon ausgehen, dass die Fachgesellschaften weiterhin die CABG als bevorzugte Revaskularisierung für Patienten mit koronarer Mehrgefäßerkrankung empfehlen, und wie bisher auch Typ1-Diabetiker einbeziehen. In einem Editorial postulieren Domanski und Farkouh folgende interessante Hypothese für die Überlegenheit der Bypass-Operation: Bei schwerer koronarer Herzerkrankung sind nicht nur mehrere Gefäße, sondern auch jedes einzelne Gefäß mit hintereinander geschalteten Stenosen multifokal betroffen. Die Dilatation einer proximalen Stenose mittels PCI kann die Perfusion verbessern, Symptome beseitigen und einem Verschluss vorbeugen, das Ischämierisiko in den langen distalen Abschnitten bleibt aber unverändert. Der Bypass fördert ebenfalls die Durchblutung. Wenn sich aber ein Verschluss proximal der Anastomose ereignet, sind die distalen Abschnitte vor einer Ischämie geschützt.

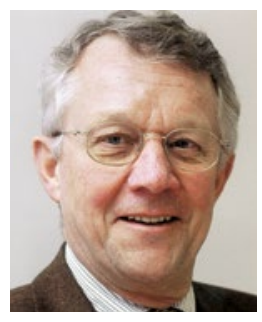

Prof. Dr. med. Heinrich Holzgreve

Internist, Kardiologische Praxis

Burgstr. 7, 80331 München

heinrich.holzgreve@t-online.de 